\title{
BMJ Open Attachment-based compassion therapy and adapted mindfulness-based stress reduction for the treatment of depressive, anxious and adjustment disorders in mental health settings: a randomised controlled clinical trial protocol
}

\author{
Jesus Montero-Marin, ${ }^{1}$ Carlos Collado-Navarro, ${ }^{2}$ Mayte Navarro-Gil, ${ }^{1}$ \\ Alba Lopez-Montoyo, ${ }^{1}$ Marcelo Demarzo, ${ }^{3,4}$ Paola Herrera-Mercadal, \\ Alberto Barcelo-Soler, ${ }^{1,5}$ Javier Garcia-Campayo ${ }^{1,5}$
}

To cite: Montero-Marin J, Collado-Navarro C, NavarroGil M, et al. Attachment-based compassion therapy and adapted mindfulness-based stress reduction for the treatment of depressive, anxious and adjustment disorders in mental health settings: a randomised controlled clinical trial protocol. BMJ Open 2019;9:e029909. doi:10.1136/ bmjopen-2019-029909

- Prepublication history and additional material for this paper are available online. To view please visit the journal (http:// dx.doi.org/10.1136/bmjopen2019-029909).

Received 19 February 2019 Revised 19 June 2019 Accepted 30 August 2019

Check for updates

(c) Author(s) (or their employer(s)) 2019. Re-use permitted under CC BY-NC. No commercial re-use. See rights and permissions. Published by BMJ.

For numbered affiliations see end of article.

Correspondence to Dr Mayte Navarro-Gil; maytenavarrogi@@gmail.com

\section{ABSTRACT}

Introduction Depressive, anxiety and adjustment disorders are highly prevalent among mental health outpatients. The lack of funding for mental health problems produces inefficient results and a high burden of disease. New cost-effective group interventions aimed at treating these symptoms might be an appropriate solution to reduce the healthcare burden in mental health units. Mindfulness-based interventions (MBIs) have shown significant reductions in anxious, depressive and adjustment symptomatology. Recent research highlights the influence of compassion as a key mechanism of change. However, MBls only address compassion implicitly, whereas compassion-based protocols consider it a core aspect of psychotherapy. In this randomised controlled trial, we hypothesise that the provision of attachment-based compassion therapy $(\mathrm{ABCT})$, which is a compassion-based protocol, will be more effective than mindfulness-based stress reduction (MBSR), which is a conventional MBI programme, for the treatment of depressive, anxious and adaptive symptoms in patients in mental health settings. Methods and analysis Approximately 90 patients suffering from depressive, anxious or adjustment disorders recruited from Spanish mental health settings will be randomised to receive 8 weekly 2 hours group sessions of ABCT, 8 weekly 2.5 hours group sessions of adapted MBSR (with no full-day silent retreat) or treatment as usual (TAU), with a 1:1:1 allocation rate. Patients in the ABCT and adapted MBSR groups will also receive TAU. The main outcome will be general affective distress measured by means of the 'Depression Anxiety Stress Scales-21' at post-test as primary endpoint. Other outcomes will be quality of life, mindfulness, selfcompassion and the use of healthcare services. There will be a 6-month follow-up assessment. Intentionto-treat analysis will be conducted using linear mixed models. Per-protocol and secondary outcome analyses
Strengths and limitations of this study

This study is the first to compare the effectiveness of an attachment-based compassion therapy (ABCT) group programme and a mindfulness-based stress reduction (adapted MBSR) group programme to treat depressive, anxious and adjustment symptoms in mental health settings.

- The inclusion of a suitable active control group and follow-up measurements will allow mediation analyses to assess potential mechanisms of improvement-such as mindfulness and self-compassion-that might function differentially in each meditation group.

- This pragmatic design includes a cost-effectiveness evaluation of both ABCT and adapted MBSR compared with the treatment as usual that could assist decision-making processes to reduce the healthcare burden in Spanish mental health units.

- The main limitation of this study is that it will not be statistically powered to detect small size differences between the groups. However, it will serve as a guide for the planning of larger, more comprehensive and more complex studies.

will be performed. A data monitoring committee comprising the trial manager, the ABCT and MBSR teachers and an independent clinical psychologist will monitor for possible negative side effects.

Ethics and dissemination Approval was obtained from the Ethics Committee of the General University Hospital of Castellón, Spain. The results will be submitted to peer-reviewed specialised journals, and brief reports will be sent to participants on request.

Trial registration number NCT03425487 


\section{INTRODUCTION}

\section{Background and rationale}

Depression is the most prevalent cause of disability and a major contributor to the disease burden worldwide. ${ }^{1}$ Its prevalence has increased in recent years, ${ }^{2}$ with the number of people living with depression around the world estimated at 322 million in $2015,4.4 \%$ of the global population. ${ }^{3}$ Between 2005 and 2015, the percentage of people suffering from depression increased by $18.4 \%$. Depression is characterised by feelings of sadness, guilt, loss of interest or pleasure, low self-worth, disturbed sleep or appetite, tiredness and poor concentration. ${ }^{3}$ These symptoms impair an individual's ability to cope with daily life and are relevant determinants of quality of life and survival because at their most severe, they can lead to autolytic behaviours. ${ }^{45}$ It has been estimated that 264 million people, $3.6 \%$ of the global population, ${ }^{3}$ suffer from a range of anxiety disorders. Again, this estimate is increasing owing to population growth and ageing processes. Anxiety refers to a group of mental disorders characterised by excessive worry and fear. ${ }^{6}$ The duration of symptoms typically experienced by people with anxiety makes it more of a chronic than episodic disorder, producing considerable impairment, decreased levels of health-related quality of life, greater healthcare utilisation and a considerable economic burden on society. ${ }^{78}$ Comorbidity between depressive and anxious symptomatology is common and is related to higher role impairment and symptom severity. ${ }^{910}$ Of patients diagnosed with major depression, $57.5 \%$ also met the criteria for at least one anxiety disorder, ${ }^{11}$ and $59.0 \%$ of individuals suffering from generalised anxiety disorder fulfil the criteria for major depression. ${ }^{12}$ Finally, adjustment disorder is a maladaptive reaction to an identifiable psychosocial stressor. ${ }^{13}$ It is among the most frequently diagnosed mental disorders, ${ }^{14-16}$ but data on its prevalence are limited. A multinational study, including Norway, Finland, Ireland and Spain, obtained a range in the general population of $0.2 \%-1 \%{ }^{17}$; in primary care, the figure was $2.9 \%$ in Spain and $9.2 \%$ in Sweden. ${ }^{18} 19$ Adjustment disorder can be persistent over time, is usually presented with depressed mood or anxiety ${ }^{2021}$ and has also been linked to suicidality. ${ }^{22}$

The consequences of these disorders are huge in terms of lost health. Given the high prevalence of these mental disorders and the low proportion of patients receiving evidence-based treatment, possibly due to educational, financial and organisational barriers, ${ }^{23} 24$ among others, it is essential that cost-effective interventions to treat these conditions become widely available. ${ }^{25}$ There is evidence that psychological treatments-specifically those framed within cognitive-behavioural therapy (CBT) - are effective in the treatment of depression, anxiety and adjustment disorders, but they are expensive when individually supplied. $^{26-28}$ Mindfulness-based interventions (MBIs) constitute a new group of psychological treatments that are immersed in the so-called 'third wave' CBTs. ${ }^{29} \mathrm{MBIs}$ endeavour to train the mind to adopt a non-judgemental present-focused awareness by cultivating full attention through formal meditation, informal practices, psychoeducation and different introspective exercises. In general, meditation can be conceptualised as a set of attention-based, regulatory and self-inquiry training regimens cultivated for various ends, including well-being and psychological health. ${ }^{30}$ There are different MBIs for the treatment of psychological disorders, although the two protocols with the greatest extension and evidence are mindfulness-based stress reduction (MBSR) ${ }^{31}$ and mindfulness-based cognitive therapy (MBCT). ${ }^{32}$ MBIs have demonstrated significant reductions in depressive, anxious and stress symptomatology, ${ }^{33}$ significant improvements in quality of life, and a lower risk of relapses in depression. ${ }^{34}$ MBIs can show efficacy similar to that of the usual CBT programmes and even to that of pharmacological treatments for anxiety and depression, with moderate effects when compared with waitlist controls but low effects when compared with other active treatments. ${ }^{28} 35$ Doubts have also recently emerged in terms of their longterm effect duration. ${ }^{36} 37$

Compassion meditation techniques are also classified under the 'third wave' psychotherapy umbrella. ${ }^{38}$ Although the concept of compassion is very recent in psychology, there is an incipient line of research that highlights its influence on psychological well-being. In general, compassion forms a particular orientation of mind that is shaped in a motivational system orientated to certain sensitivity to suffering and a commitment to relieve suffering by recognising the universality of pain and the ability to meet that pain with equanimity. ${ }^{39}$ Compassion for the self (self-compassion) is this attitude focused on the self, ${ }^{41}$ and it has been found to be a key mechanismtogether with mindfulness abilities-in the effectiveness of techniques such as MBSR and MBCT. ${ }^{42} 43$ Authors have hypothesised that self-compassion could be the 'crucial attitudinal factor' in the relationship between mindfulness and mental health. ${ }^{44}$ However, this construct only works implicitly in the MBI protocols. A strong current of authors has recently been defending the need to work explicitly on compassion, considering it the real engine of change in MBIs. Currently, several protocols expressly focus on cultivating compassionate attitudes, for instance, the 'Mindful Self-Compassion Programme', ${ }^{45}$ with a general approach based on self-compassion skill building; 'Compassionate Mind Training', ${ }^{46}$ with a general clinical approach and 'attachment-based compassion therapy' (ABCT), ${ }^{47}$ which can be adapted to the healthcare environment ${ }^{48}$ and is applicable to general and clinical populations. ${ }^{49} 50$ Moderate inverse relationships between self-compassion and depression, as well as anxiety and stress symptoms, have been observed, ${ }^{40}$ and compassion programmes such as ABCT have produced large effect sizes (ESs) when treating depressive and anxious symptomatology in fibromyalgia patients, compared with active controls. $^{51}$

If the self-compassion construct is at the very base of the effectiveness of MBIs when treating depressive, 
anxiety and stress symptomatology, we could suppose that a specific compassion-based protocol might be more effective for treating people who suffer from those symptoms than a conventional MBI. No previous studies have directly compared specific compassion protocols such as ABCT with conventional MBIs such as MBSR, although previous research examining the outcomes of each group of programmes independently might suggest that explicitly teaching self-compassion to specifically increase self-compassion levels, as is done in the ABCT programme, would make a difference. ${ }^{42} 49$

\section{Objectives}

The main aim of this study is to assess the effectiveness of ABCT added to treatment as usual ('ABCT+TAU'), compared with MBSR added to TAU ('MBSR + TAU'), in patients from mental health settings suffering from depressive, anxiety or adjustment disorders. Secondary aims will be (1) to compare 'MBSR +TAU' and 'ABCT+TAU' with the 'TAU alone'; (2) to analyse the possible mediating role of mindfulness and self-compassion on improvements in the 'MBSR + TAU' and 'ABCT+TAU' groups compared with 'TAU alone' and (3) to evaluate the cost-effectiveness of both 'MBSR +TAU' and 'ABCT+TAU' groups compared with 'TAU alone'.

The principal hypothesis is that 'ABCT + TAU' will be more effective than 'MBSR +TAU' for treating depressive, anxiety and adjustment disorders in patients in mental health settings. Secondary hypotheses are: (1) 'MBSR +TAU' and 'ABCT +TAU' will be more effective than 'TAU alone' for treating depressive, anxiety and adjustment disorders in patients in mental health settings; (2) mindfulness will be a mediator of the 'MBSR + TAU' programme improvements, whereas self-compassion will be a mediator of the corresponding 'ABCT +TAU' and (3) 'ABCT + TAU' will prove to be more cost-effective than 'MBSR + TAU', and both ABCT + TAU' and 'MBSR +TAU' programmes will prove to be more cost-effective than TAU alone.

\section{Trial design}

This study is a multicentre, randomised controlled trial (RCT) comprising three parallel arms, with pretreatment, post-treatment and 6-month follow-up measurements, and a 1:1:1 allocation rate among groups. ${ }^{52}$ Patients from three mental health units in Castellón, Spain will be randomly assigned to three different parallel conditions, with two psychological intervention groups ('ABCT+TAU' and 'MBSR+TAU') and the usual psychological/psychiatric treatment managed by their specialist ('TAU alone'), to test the superiority of 'ABCT+TAU' provision compared with 'MBSR+TAU', but also of 'ABCT+TAU' or 'MBSR+TAU' compared with the 'TAU alone' provision. Those patients allocated to 'TAU alone' will be offered an adaptation of the mindfulness and compassion programmes after finishing the trial at the 6-month follow-up.

\section{METHODS}

This protocol was designed in accordance with the Standard Protocol Items: Recommendations for Interventional Trials 2013 statement. ${ }^{53}$

\section{Study setting}

Participants will be recruited from the mental health units of Illes Columbretes, Rafalafena and Cardenal Costa Healthcare Centres in Castellón, Spain. Psychiatrists or psychologists will be trained to use the Structured Clinical Interview for Diagnostic and Statistical Manual of Mental Disorders-Fifth Edition (DSM-5) Research Version ${ }^{54}$ as a semistructured guide for making DSM-5 diagnoses, ascertaining inclusion and exclusion criteria. When the clinicians at any of these mental health units detect a potential participant, he/she will be referred to the corresponding research unit to be offered the information about the study. It will be explained to the patients that only the research team will have access to their clinical history data and nobody outside of their health centre will be able to consult their medical records.

\section{Eligibility criteria}

The following inclusion and exclusion criteria will be applied when recruiting patients. Only patients meeting the inclusion criteria will be accepted into the study.

\section{Inclusion criteria}

- Age: between 18 and 75 years.

- Depressive and/or anxious disorder, or adjustment disorder with depressive and/or anxious symptomatology, according to clinical criteria based on the DSM-5.

- Disorder of mild or moderate severity according to clinical criteria (based on DSM-5).

- Understanding of spoken and written Spanish.

- Provision of written informed consent.

Exclusion criteria

- Having done any type of meditative/contemplative practice in the previous year.

- The diagnosis of any disease that can affect the central nervous system (eg, brain pathology, traumatic brain injury and dementia).

- Other psychiatric diagnoses or acute psychiatric illness (eg, substance dependence or abuse, history of schizophrenia or other psychotic disorders, eating disorders)

- Any medical condition or infectious or degenerative disease that can affect mood.

- Presence of delusional ideas or hallucinations, whether or not consistent with mood and suicide risk.

Once informed, candidates will be asked whether they wish to participate in the study. If they accept, they will subsequently be provided with the confidentiality and informed consent documents. Those who do not accept will continue with their usual treatment at their health centre. 


\section{Patient and public involvement}

Although importance is being given to engaging stakeholders in the choice of research question, ${ }^{55}$ no patients or members of the public were directly involved in the development of the research question in this case because of the novelty and incipient use of the ABCT protocol. However, prior to the recruitment stage, a group of advisory patients who were contacted through the Depressive Disorders Association of Aragon were interviewed at the mental health units about the research question as well as the design and feasibility of the interventions. These advisers agreed with the relevance of the study question but they proposed that the initially planned day retreat session, included in the MBSR programme, should be ruled out because of likely schedule incompatibilities. All of this was corroborated by study patients, who were specifically asked for their schedule preference and the possibility of including a day retreat session. Advantage was taken of this to adjust the design in order to balance the intensity of both ABCT and MBSR interventions. Study patients were only directly involved in this part of the design. No patients or members of the public will be engaged in recruitment, conducting and outcome measurement, over which the research group will have full control, although an independent advisory reference group will be put together and meet three times during the course of the study to receive updates from the study researchers. These meetings will serve to review the study development, and the data monitoring committee (DMC) will be informed of possible comments and suggestions. Study results will be disseminated to participants by email, including a brief personal and comprehensive report on request. The burden of the interventions included in this study will be not assessed by patients, but they will provide data on the use of healthcare and social services and other economic information.

\section{Interventions}

Patients in all conditions will be informed that they will continue to receive the services as usual before enrolment in the study, so that all participants regardless of the arm to which they are assigned will receive their corresponding TAU. To be consistent with the pragmatic nature of the study, care will be taken not to interfere with routine practices for intervention delivery. Thus, ad hoc adherence optimisation procedures will not be used. Patients will be informed in the first interview that they will be free to decide whether to discontinue or modify their allocated intervention, on request, in response to possible harm, adverse events or improving/worsening symptoms, without affecting their ability to continue to receive their usual treatment. Antidepressant and/or anxiolytic medication use would be permitted if the participant agreed not to modify the pharmacological treatment that was prescribed during the study period (it would be established that the treatment could be decreased but not increased). No other different psychological and meditative treatments outside of usual treatment will be permitted to be received during the study period.

\section{Attachment-based compassion therapy}

Subjects assigned to the ABCT intervention arm will receive 8 weekly 2 hours group sessions conducted by a clinical psychologist certified in ABCT and specifically trained to ensure the integrity of the ABCT intervention, following its specific content and avoiding overlapping with other psychotherapeutic techniques. Group sizes can range from 10 to 15 participants. All sessions will be taught by the same psychologist following a standardised protocol ${ }^{48}$ to maintain consistency with the programme. The ABCT protocol includes teachings, daily simple exercises, meditation, visualisations and specific practices to augment the patients' ability to be considerate and kind towards themselves and their own experience (more specifically their experience of suffering), and others' experience of suffering. ABCT is based on attachment theory, ${ }^{56}$ which provides a framework for understanding the links between close relationships and psychopathology. Subjects will receive written and audio materials and resources with theoretical concepts, exercises and meditation guides to support home practice. Home exercises will be commented on in each session in order to resolve possible doubts and encourage adherence to the programme. Table 1 shows the session structuring in terms of theory and formal and informal practices.

\section{Mindfulness-based stress reduction}

Subjects assigned to the MBSR intervention arm will receive 8 weekly 2.5 hours group sessions conducted by a clinical psychologist certified in MBSR therapy and specifically trained to ensure the integrity of the MBSR programme, following its specific content and avoiding overlapping with other psychotherapeutic techniques. Group sizes will range from 10 to 15 participants. All sessions will be taught by the same psychologist following the standardised MBSR authorised curriculum guide $\mathrm{e}^{3157}$ to reinforce consistency with the original programme. The MBSR protocol presents an educational orientation that includes teachings, informal exercises, formal meditation (eg, sitting meditation, body scan, walking meditation and gentle yoga), as well as individual and group dialogues and inquiries about perceptions, habits and patterns that may interfere with learning. However, the practice of meditation exercises during a full day of retreat included in the original protocol will be ruled out to avoid schedule incompatibility, and thus a workable modification will be established. This same adjustment has also been done in other previous MBSR adaptations. ${ }^{589}$ No other major modifications will be made to the form or content, instructor characteristics or intention of the programme. ${ }^{60}$ As in the previous intervention, subjects will receive written and audio resources with theoretical concepts, exercises and meditation guides to support home practice. Home exercises will be commented on in each session to resolve doubts and encourage adherence. 
Table 1 Theory, formal and informal practice content of the ABCT programme

Theory: The workings of our brain; the reality of suffering: primary and secondary suffering; what is and is not compassion?

Formal practice: Compassionate breathing and compassionate body scan; compassionate coping with difficulties

Preparing ourselves for Informal practice: $3 \mathrm{~min}$ compassionate practice; self-compassion diary;

\begin{tabular}{lll} 
Session 1: & compassion. Kind attention savouring and giving thanks \\
\hline Session 2: & Discovering our compassionate Theory: Compassion and mindfulness; self-esteem and compassion; fear of
\end{tabular} world

compassion

Formal practice: The figure of affect: connecting with basic affection;

developing a safe place; The compassionate action; Identifying the figure of secure attachment

Informal practice: The object that joins us to the world; diary of compassion practice; what are we good at?

Session 3: Developing our compassionate Theory: How compassion acts; effectiveness of compassion; self-criticism world

Formal practice: Developing the figure of secure attachment; developing the compassionate voice

Informal practice: Writing a letter to a compassionate friend

$\begin{array}{lll}\text { Session 4: } & \begin{array}{l}\text { Understanding our } \\ \text { relationships with compassion }\end{array} & \begin{array}{l}\text { Theory: Attachment styles; importance of these styles in everyday life } \\ \text { Formal practice: Becoming aware of our attachment style; ability to receive } \\ \text { affection: friend, indifferent person, enemy } \\ \text { Informal practice: Letter to your parents }\end{array}\end{array}$

Session 5: Working on ourselves

Theory: Importance of affection towards ourselves and others

Formal practice: Showing affection to friends and indifferent people; showing affection to ourselves; reconciliation with our parents; three positive aspects and three negative aspects of our parents

Informal practice: The greatest display of affection (in general and from our parents); the illusion of labels $\begin{array}{ll}\text { Session 6: } & \text { Advanced compassion (l): } \\ \text { forgiveness }\end{array}$

Theory: Guilt and the importance of forgiveness

Formal practice: Forgiving yourself; asking others for forgiveness; forgiving others and showing compassion to enemies; showing forgiveness for the hurt caused by loved ones (only for people with this experience); recapitulation Informal practice: Compassion in everyday life; interdependence

Session 7: Advanced compassion (II): becoming your own attachment figure and handling difficult relationships

\section{Session 8: Beyond compassion:} equanimity
Theory: Working in three periods; Envy; usefulness of being our attachment figure; difficult people

Formal practice: Working with envy; becoming our own attachment figure; handling difficult relationships

Informal practice: Not taking anything personally; looking others in the eye and connecting with their suffering

Theory: Equanimity; How to keep up the practice of compassion for a lifetime Formal practice: Equanimity (I): We are all equal; Equanimity (II): The illusion of categories; Equanimity (III): Showing the world the gratitude we have not been able to give back

Informal practice: Our values and their relationship with compassion; The cosmic attachment figure; The tantric embrace

ABCT, attachment-based compassion therapy.

The theory, practice and support contents that form the structuring of the sessions are described in table 2.

\section{Treatment as usual}

TAU is delivered in current daily practice by psychiatrists and clinical psychologists of the national mental health service in Spain. TAU can refer to psychiatric treatment, which typically includes prescription and monitoring of antidepressant and/or anxiolytic medication, psychological treatment (possibly including case management, CBT techniques, empathic listening and/or supportive counselling), or a combination of both treatments. Frequency of visits will vary depending on the type of treatment received and the severity of the case, but patients' visits to their specialist are typically scheduled at intervals of at least 1 month.

\section{Outcomes}

Several outcomes will be assessed and compared between the psychotherapy arms and the 'TAU alone' condition to evaluate the effects and cost-effectiveness of the programmes. Following the tripartite model of 
Table 2 Theory, practice and support contents of the adapted MBSR programme

Theory: When we are breathing, there are more positive things than negative things inside us, whatever the problems we must face; formal and informal meditation

\section{Introduction: What is}

\begin{tabular}{ll} 
Session 1: & Mindfulness and MBSR? \\
\hline Session 2: & $\begin{array}{l}\text { Body awareness and } \\
\text { perception }\end{array}$ \\
Session 3: & $\begin{array}{l}\text { Get out of autopilot and be in } \\
\text { the present }\end{array}$
\end{tabular}

Practice: Attention to breathing here and now; body exploration meditation support: raisin meditation.

Theory: What glasses are we wearing? How we see and interpret situations determines how we respond to them; perception and conditioned patterns Practice: breathing meditation/yoga Support: 9 dots exercise

Theory: We are on the path of consciousness; doing mode versus being mode; thoughts (introduction) Practice: sounds meditation

Support: diary of pleasant events

\section{Session 5: Acceptance}

Theory: Curiously investigate reactive patterns; stress physiology: sympathetic/ parasympathetic system; physiology of emotions

Practice: name emotions/allow emotions Support: diary of unpleasant events

\section{Theory: Midpoint of the course: renewing the commitment; opening a space} between the stimulus and the reaction; mechanisms of coping with stress Practice: standing and walking meditation/attention in open focus Support: response versus reaction exercise

$\begin{array}{ll}\text { Session 6: } & \begin{array}{l}\text { Interpersonal relationships/ } \\ \text { letting go of difficult situations }\end{array} \\ \text { Session 7: } & \begin{array}{l}\text { Integrate the practice of } \\ \text { Mindfulness in everyday life }\end{array}\end{array}$

Session 8: Renew our intention, self-care and self-compassion
Theory: Judgement, comparison and presumptions; The alteration produced by conflicting communication; letting go of difficult situations (ruminations) Practice: Meditation on letting go of difficult situations/aikido Support: active listening exercise; diary of difficult or stressful conversations

Theory: Self-care; reflection on nutritive and adaptive lifestyles versus maladaptive, self-limiting and self-destructive; become aware of our values; How I position myself before what happens to me according to my values Practice: Metta Meditation. Loving kindness

Support: Exercise of conscience: why am I here?

Theory: Self-care and how to grow it; Collective wisdom: what have we learnt, from ourselves, from others? We are different now with respect to when we arrived.

Practice: Meditation Cultivating Self Compassion; Mountain Meditation Support: Dyadic exercise: who I am? Why I am here? Where do I want to go?

MBSR, mindfulness-based stress reduction.

anxiety, depression and distress, which suggests that both anxiety and depression conditions have in common several symptoms of negative emotional states, ${ }^{61}$ the primary outcome will be a self-reported global measure of general affective distress to provide data on intervention effectiveness. Secondary outcomes will permit the development of a more detailed exploration into how psychotherapy arms work in terms of health-related quality of life, and mindfulness and self-compassion as possible mediators of psychotherapy effects, as well as the use of healthcare and social services associated with conditions. Assessors will be specifically trained to administer all the referred instruments. Outcomes are summarised in table 3 .

\begin{tabular}{lllll}
\hline Table 3 & Study outcomes & & & \\
\hline Outcome & Area & Source & Variable type & Time \\
\hline DASS-21 & General affective distress & Self-reported & Treated as interval & Baseline, post-treatment and follow-up \\
EQ-5D & Health-related quality of life & Self-reported & Treated as interval & Baseline, post-treatment and follow-up \\
FFMQ & Facets of mindfulness & Self-reported & Treated as interval & Baseline, post-treatment and follow-up \\
SCS & Facets of self-compassion & Self-reported & Treated as interval & Baseline, post-treatment and follow-up \\
CSRI & Health and social services use & Self-reported & Treated as interval & Baseline and follow-up \\
\hline
\end{tabular}

CSRI, Client Service Receipt Inventory; DASS-21, Depression Anxiety Stress Scales; EQ-5D, European Quality of Life Scale-5 Dimensions; FFMQ, Five Facet Mindfulness Questionnaire; SCS, Self-Compassion Scale. 


\section{Main outcome}

The main effectiveness outcome will be general affective distress, which will be assessed through the brief version of the 'Depression Anxiety Stress Scales-21' (DASS-21). ${ }^{62}$ The DASS-21 is a self-report questionnaire composed of the three negative affectivity subscales of depression, anxiety and stress. Each subscale is made up of seven items, and each item comprises a statement and four Likert-type response options to reflect severity (rated from 0 'did not apply to me at all' to 3 'applied to me very much, or most of the time'). To yield equivalence with the long DASS-42 version, the total score of each subscale is doubled. ${ }^{62}$ In addition, this questionnaire provides us with a one-dimensional global measure of emotional distress which will be considered the main study outcome. It comprises all 21 items configuring a general factor, and it will be used as a continuous dimensional variable that ranges from 0 to 126. Participants are asked to indicate how much each statement applied to them over the past week. The DASS-21 has been specifically validated in the Spanish population, showing strong indices of internal consistency (total scale $\alpha=0.96$; depression $\alpha=0.93$; anxiety $\alpha=0.86$ and stress $\alpha=0.91$ ) and expected patterns of discriminant, convergent and factorial validity. ${ }^{63}$

\section{Secondary outcomes}

A general health-related quality-of-life measurement will be performed to compare possible differences between treatments. The 'European Quality of Life Scale-5 Dimensions' (EQ-5D) ${ }^{64}$ will be used. The EQ-5D is a standardised instrument to measure quality of life that has been applied to a wide range of health conditions and treatments, and provides a simple descriptive profile and a single index value for general health status. It includes information about mobility, personal care, daily activities, pain/discomfort, anxiety/depression and a personal estimate of the present general health state compared with the previous 12 months, all of them rated on a Likerttype scale with three response options. In addition, this questionnaire includes a visual analogue scale (VAS) to measure present health state, with all the possible values ranging from 0 to 100 . The Spanish version of the EQ-5D will be used. ${ }^{65}$ The ability of the descriptive system and the VAS of the Spanish version of the EQ-5D to discriminate between healthy individuals and critical and chronic patients has been observed to be good.

As previously mentioned, mindfulness and self-compassion have been presented as possible mechanisms underlying the therapeutic effects of meditative interventions. ${ }^{42} 43$ Thus, to evaluate their possible differential mediation effect between treatments, they will be measured using the 'Five Facet Mindfulness Questionnaire' (FFMQ $)^{66}$ and the 'Self-Compassion Scale' (SCS) ${ }^{67}$ The FFMQ is a 39-item self-report measure of mindfulness. It comprises five facets: observing, describing, acting with awareness, non-judging of inner experience and non-reactivity to inner experience. Respondents indicate on a 5-point Likert-type scale the degree to which each item is true for them (ranging from 1 'never or very rarely true' to 5 'very often or always true'). Higher scores reflect higher levels of mindfulness. The facet scores can be combined to produce a total mindfulness score that ranges from 39 to 195 . The Spanish version of the FFMQ $^{68}$ has shown good psychometric properties, with Cronbach's alpha values between 0.80 and 0.91 on all the subscales. On the other hand, the SCS is a 26-item questionnaire designed to assess the components of self-compassion across the facets of common humanity, mindfulness and self-kindness. The SCS uses a 5-point Likert-type scale, ranging from 1 ('almost never') to 5 ('almost always'). Higher scores indicate higher levels of self-compassion. Although the construct was defined using three facets, factor analysis suggests six subscales, which represent the positive and negative aspects of each facet as follows: self-kindness, self-judgement, common humanity, isolation, mindfulness and overidentification. The subscale scores can be combined to produce a total self-compassion score ${ }^{69}$ that ranges between 26 and 130. The Spanish version of the $\mathrm{SCS}^{70}$ is a valid and reliable instrument, with internal consistency values $\alpha \geq 0.72$, and test-retest reliability values $\geq 0.81$ on all the subscales.

The possible differential use of healthcare and social services and other economic effects (eg, time corresponding to sickness absence) between treatments will be evaluated by means of the "Client Service Receipt Inventory' $(\mathrm{CSRI})^{71}$ questionnaire. The version of the CSRI questionnaire to be used in this study has been designed to collect retrospective data on service utilisation in the 8 months prior to baseline assessment and in the 8 months prior to follow-up measurement. The Spanish-validated version of the CSRI will be used. ${ }^{72}$

In addition to the previously mentioned outcomes, the following sociodemographic data will be collected at baseline to describe and compare the general characteristics of participants included in the different study arms: age, gender, marital status (single, married/relationship, separated/divorced, widowed), number of children, place of residence (own home, relative's home, neighbour's/ friend's home, residential facility or other), education (no studies, primary studies, secondary studies, university studies or other), employment (unemployed, temporary contract, permanent contract, self-employed, home duties, student, benefit recipient or retired), economic level (<national minimum wage (NMW) or NMW, $1-2 \mathrm{x}$ NMW, 2-4 x NMW or $>4 \times$ NMW).

All the baseline information concerning outcomes (table 3) and sociodemographic variables will be collected before randomisation. Data on attendance at MBSR and ABCT groups will be recorded. Moreover, participants in meditation groups will be asked at the post-test about the number of weekly meditation practice days, the number of minutes of meditation on each practice day, and what they have learnt and taken from the group (as an open question to be completed in approximately three lines). All the participants will provide post-treatment data about mental healthcare (continues TAU, 
abandons TAU, discharged, other) and possible changes in pharmacological treatment in the previous 2 months (no change, reduced medication, increased medication, change of medication). Finally, participants in meditation groups will be asked again at the 6-month follow-up about the number of weekly meditation practice days and the number of minutes of meditation on each practice day. At follow-up, all participants will also provide data about mental healthcare (continues TAU, abandons TAU, discharged, other), and possible changes in pharmacological treatment in the last 6 months (no change, reduced medication, increased medication and change of medication).

\section{Sample size}

Sample size has been estimated considering the main hypothesis ('ABCT+TAU' will be more effective than 'MBSR +TAU' for treating depressive, anxiety or adjustment disorders). There are no references on previous direct comparisons between ABCT and MBSR groups, so we will perform an exploration in this respect. According to the literature and considering the TAU active control comparator used, we assume a large effect of ABCT and a small effect of MBSR in the primary outcome of general affective distress. ${ }^{28} 365173$ Therefore, with an equal 1:1:1 allocation rate, supposing a large difference between ABCT and MBSR conditions, with an ES of roughly $\mathrm{d}=0.80$, accepting an alpha of 0.05 and a beta risk $<0.2$ in a bilateral contrast, we will need approximately 75 patients: 25 in the MBSR condition, 25 in the ABCT group and 25 in the TAU condition. Assuming a patient attrition rate at follow-up of approximately $15 \%-20 \%$, the total sample size needed will be established at 90 patients ( 30 in each group).

\section{Recruitment}

Recruitment will be conducted consecutively in one wave from September 2018 until the target sample size is completed (patient recruitment is expected to be finalised in February 2019). Figure 1 shows the participant timeline overview and figure 2 is a more detailed study flow chart showing recruitment through to data collection and results. We met with the psychologists and psychiatrists responsible for three mental health units in Castellón and gave them verbal and detailed written information about the study and about the selection criteria of the potential participants. Having agreed to collaborate with us, they will oversee the informing of patients who meet the established selection criteria and the referring of those patients who agree to participate to the research group assessors at the corresponding mental health unit.

After an (approximately 1 hour) individual interview with the patients to inform them about the study, in which they are also told about the importance of commitment in these types of interventions, all those who agree to participate, provide written informed consent and complete the baseline assessment, will be included in order to be randomised. During this interview, patients will be able to ask questions on possible doubts about any aspect of the study, which will be answered by the assessors. Nonetheless, all participants must agree to their inclusion in the study prior to randomisation, and they will not be informed of their group allocation until after completion of the baseline measurements.

\section{Randomisation and blinding}

A simple random sequence will be remotely generated after baseline evaluation by a member of the research group who is not involved in the study and has no knowledge of the study aims, using computer-generated random sequence numbers with a 1:1:1 allocation rate. Participants will be informed by telephone of the experimental condition to which they have been randomly assigned, that is, to 'ABCT+TAU', 'MBSR + TAU' or 'TAU alone'. The numeric random sequence document will be unavailable to the researcher in charge of enrolling and assessing participants, in order to avoid predictability. He will be unaware of the treatment group to which any patient belongs and will be advised not to

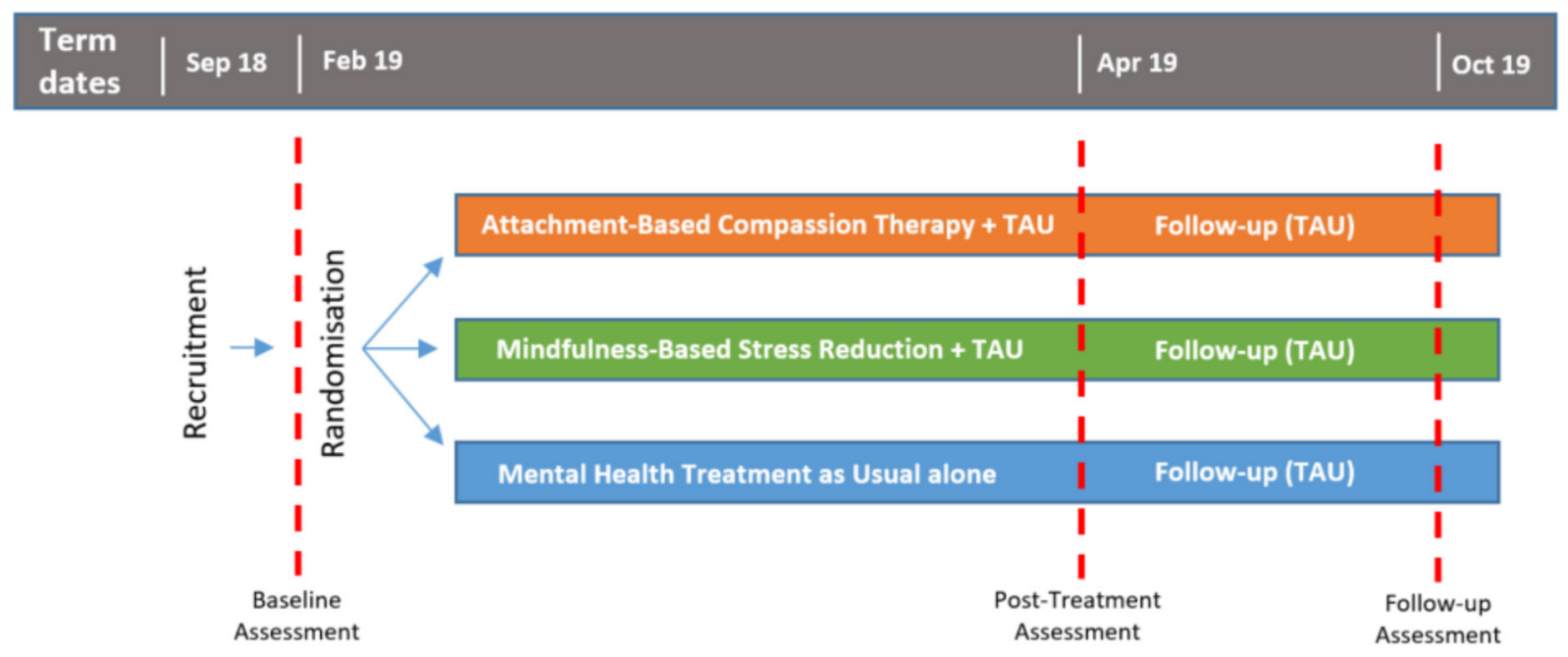

Figure 1 Partcipant timeline. TAU, treatment as usual. 


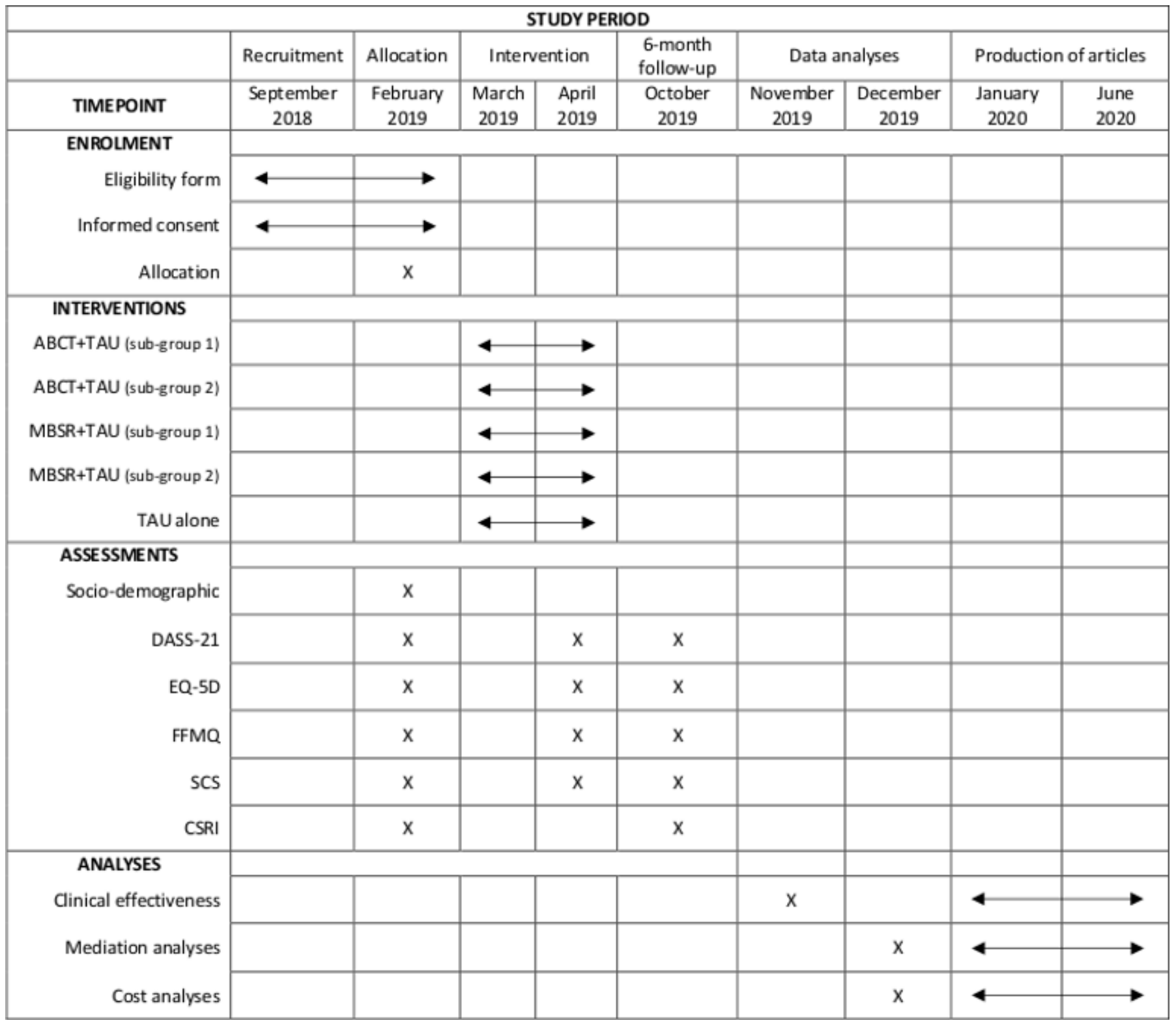

ABCT+TAU (Attachment-Based Compassion Therapy plus Treatment as Usual); MBSR+TAU (Mindfulness-Based Stress Reduction plus Treatment as Usual), TAU alone (Treatment as Usual alone); DASS-21 (Depression Anxiety Stress Scales); EQ-5D (European Quality of Life Scale); FFMQ (Five Facet Mindfulness Questionnaire); SCS (Self-Compassion Scale); CSRI (Client Service Receipt Inventory).

Figure 2 Study flow.

ask for this information. The psychologists and psychiatrists providing TAU will also be unaware, for as long as possible, of the arm to which the patient has been randomised. Patients will be requested to not discuss the intervention they are receiving with their corresponding clinicians. Participants and group instructors cannot be blind to arm allocation because of the nature of the interventions, which are based on psychotherapeutic content that can be identifiable-although all the study arms will configure active groups of treatment. However, primary analysis will be conducted by a statistician who will receive the database blind to which arm comprises each intervention, and to any other sociodemographic information that might facilitate identification of the intervention group. The groups to which participants are allocated may only be unblinded to the trial manager in the event of reported adverse events or other unintended effects of interventions or trial conduct.

\section{Inducements for participation}

Participants will not receive any remuneration for taking part in the study, and there will be no inducements for completing the interventions beyond their suffering from depression, anxiety or adjustment disorders that must be treated. However, through the individual interview prior to randomisation, participants will be strongly advised of the importance of engagement in their corresponding programmes and practices so that the interventions are able to work and thus to be relieved of their symptoms. Moreover, regular motivational techniques to increase 
adherence of participants to sessions and daily tasks are included in the own programmes-for example, daily tasks aids, such as compassion or mindfulness meditation audio recordings (depending on the condition) will be provided in each group, and all participants will be encouraged to do a daily task at home and will be asked to keep a daily written record of the number/duration of practice sessions. To promote participant retention and completion of the assessment, those participants who complete all the measurement waves will be given the opportunity on completion of the trial to receive information concerning the evolution of their affective distress scores throughout the study, and to receive a final report on request.

\section{Data management}

To ensure the quality of the data, assessors will be specifically trained and the transcription processes from the paper and pencil surveys to the electronic data sheets will be performed twice by two independent researchers, while a third person will integrate them by revising the original source in case of discrepancy. This third researcher will also perform range checks for data values. Only electronic data will be used for the study. The data will be treated anonymously and will be stored on a computer server at the research group's headquarters; paper and pencil questionnaires will be kept under lock and key in a filing cabinet at the research group's headquarters. Data from outcome measurements will be separated from any identifying information, and advanced encryption standard strategies concerning data encryption and use of personal passwords will be implemented to the database to guarantee protection of personal information.

\section{Analysis plan}

Sociodemographic and outcome descriptive data at baseline will be analysed by means of frequencies (percentages), medians (IQR) and means (SD) according to their level of measurement and statistical distribution. The three treatment conditions will be compared with evaluate the success of randomisation, including diagnosis, by using the $\chi^{2}$ or Fisher's exact test for qualitative variables, and Kruskal-Wallis or one-way analysis of variance tests depending on the shape of the distributions of outcome measures.

\section{Clinical effectiveness}

Reporting of this RCT will follow the recommended Consolidated Standards of Reporting Trials guidelines. ${ }^{745}$ Primary analysis will consist of a comparison between 'ABCT+TAU' and 'MBSR + TAU' arms at post-test as primary endpoint, adjusted for those routine sociodemographic variables that could be unbalanced at baseline, and considering the main outcome DAAS-21 total score as a continuous variable. Analysis will be performed using a repeated measures design with an intention-totreat base and linear mixed-effects regression. Subjects will be introduced in the random part of the model by means of the restricted maximum likelihood method, which produces unbiased estimates of variance and covariance parameters when using small sample sizes or unbalanced data. ${ }^{76}$ The 'treatment by time' interaction will be specifically considered to assess the trajectories throughout the study and to determine whether possible differences between arms will be consistent over time. Raw estimations of slope coefficients (and their 95\% CI) will be calculated. Cohen's d statistic, as an ES measure of between-group differences, will be calculated by means of pooled pretest SDs to weight the differences in the pre-post marginal means-using SEs to derive SD and considering the size of the groups. ${ }^{77} \mathrm{ES}$ values of $\mathrm{d}=0.20$ are considered small, with 0.50 medium and 0.80 large. ${ }^{78}$ In addition, marginal $R^{2}$ values (only considering the variance of the fixed effects) and conditional $R^{2}$ values (considering both the fixed and the random effects) will be calculated. ${ }^{79}$

Secondary analysis will evaluate the maintenance of the improvements achieved at 6-month follow-up. Moreover, other secondary analyses will explore the clinical significance of improvements using the Jacobson and Truax method ${ }^{80}$ to establish both the cut-off point and reliable change index on the DAAS-21 total score. This criterion of classification will be used to calculate absolute risk reduction and number needed to treat (NNT); a 95\% CI for each NNT will be calculated. We will also explore results of the subscales of the DAAS-21 and the secondary outcomes of EQ-5D, FFMQ and SCSconsidering total scores and subscales-using the same analytical strategy used for the primary analysis. Sensitivity analysis will be performed to assess the effects of missing data, which will be replaced by multiple imputations based on chained equations and will include the main and secondary outcomes at all the waves-as well as routinely collected sociodemographic data-as long as there are $<40 \%$ missing data in the corresponding outcomes to ensure validity, and whether missing values are distributed at random(MAR). ${ }^{81}$ A per-protocol analysis will also be conducted, considering the criterion of $\geq 50 \%$ attendance of sessions as the minimum dose assumed to reach programme compliance. ${ }^{43}{ }^{82}$ We will also perform a parallel evaluation of the effect for each session attended separately. Predefined subgroup analyses will also be performed on the primary outcome using interaction tests ${ }^{83}$ by (1) baseline DAAS-21-those patients that are initially worse might drive change; (2) symptomatology (eg, depression, anxiety, mixed and adjustment disorder)—some programmes could function better with specific diagnoses and (3) amount of home practice-one might expect that the more home practice, the greater the benefits. Multilevel models will also be used to assess the non-specific, that is, common, ingredients of MBSR and ABCT compared with TAU by adding the treatment group in the mixed model random part and to assess their transdiagnostic effectiveness by including the diagnosis group in this part. 


\section{Mediation analyses}

The potential role of mindfulness and self-compassion as possible mediators of changes will be explored on the primary and secondary outcomes. For this purpose, (1) primary and secondary outcome prefollow-up differential scores will be calculated and considered dependent variables; (2) pre-post differential scores of mindfulness and self-compassion total scores-as two measures composed by the sum of their corresponding facets-will be calculated and included in the same model as parallel process variables; (3) the group condition (with two independent possibilities: 'ABCT+TAU' vs 'TAU alone' or 'MBSR+TAU' vs 'TAU alone') will be considered the independent variable. The mediating analyses will be conducted using maximum likelihood-based path analyses for continuous dependent variables. Regression coefficients of bootstrapped indirect effects will be estimated, as well as their 95\% CIs based on 10000 bootstrap samples, considering a significant mediating effect when the referenced 95\% CI does not include zero. ${ }^{84}$ Total or partial mediation will be established by calculating the percentage of the total mediating effects and the percentage of each mediator independently.

\section{Cost analyses}

The economic evaluation of this study will follow the guidelines for conducting pharmacoeconomic analyses in the Spanish context, the Consolidated Health Economic Evaluation Reporting Standards, ${ }^{85}$ as well as the guidelines set by the International Society for Pharmacoeconomics and Outcomes Research. ${ }^{86}$

Cost will be estimated from the healthcare and societal perspectives for the 8 months prior to baseline and during the 8 months prior to follow-up. The cost derived from pharmacological therapy, medical tests and the use of health-related services will be added to calculate direct healthcare costs using the unit costs of the corresponding financial year in $€$. The cost of medication will be calculated by determining the price per mg according to the information of the drug from the Spanish Ministry of Health, Consumer Affairs and Social Welfare, including value-added tax. Total costs will be estimated by multiplying the mg price by the daily dosage in $\mathrm{mg}$ and the number of days of treatment. The unit cost data for medical tests and health services will be obtained from the OBLIKUE database of healthcare costs. ${ }^{87}$ Indirect costs (eg, lost productivity) will be estimated using the human capital approach that involves multiplying the minimum daily wage in Spain for the corresponding year by the number of sickness absence days. Finally, total costs will be calculated by summing direct and indirect costs and will be expressed in $€$.

The effectiveness of the interventions will be estimated by means of the difference between the DAAS-21 score at baseline and at follow-up, and utility will be estimated using quality-adjusted life-years (QALYs) at follow-up. Cost-effectiveness will be analysed through the estimation of incremental cost-effectiveness ratios (ICERs) for the intervention groups of 'ABCT+TAU' and 'MBSR +TAU', with 'TAU alone' considered a control group. ICER is the ratio between incremental costs and incremental effectiveness and is calculated as 'cost active intervention group-cost control intervention group/utility active intervention group-utility control intervention group'. In addition, to provide an estimation of the extra costs that are needed to gain one responder with clinical significance in 'ABCT+TAU' and in 'MBSR+TAU' compared with 'TAU alone', we will perform cost-effectiveness analysis considering a dichotomous treatment response outcome based on the Jacobson and Truax classification criterion referenced above. ${ }^{80}$ Cost-utility will be analysed through the estimation of incremental cost-utility ratios (ICURs) as the ratios between incremental costs and incremental utilities measured in QALYs. QALYs will be estimated using the area-under-the-curve technique. ICURs will be estimated using the same equation but employing QALYs instead of clinical units.

Two cost-utility planes will be plotted, one for each contrast ('MBSR+TAU' vs 'TAU alone' and 'ABCT+TAU' vs 'TAU alone'). In each cost-utility plane, the incremental costs between the corresponding intervention group and the 'TAU alone' group will be plotted on the $y$-axis, and the incremental utility will be plotted on the $\mathrm{x}$-axis.

\section{Level of significance}

The overall alpha level will be set at 0.05 . No corrections for multiple comparisons will be made for the primary analysis-because only one contrast will be made in this regard, that is, 'ABCT + TAU' vs 'MBSR + TAU' at post-test as primary endpoint-and for the secondary analysesbecause they will be considered explorative in the general context of the present study. ${ }^{88}$

\section{Statistical analysis plan}

A statistical analysis plan (SAP), including a comprehensive and detailed description of the methods and presentation of data analysis, will be developed during the course of the study. The SAP will be finalised by a designated statistician (JM-M) with appropriate qualifications and experience, and will be revised by all the study team members prior to the database lock for use in the final statistical analysis. Any discrepancies or changes made between the analysis plan in the present protocol and final SAP will be explained in the SAP document.

\section{Monitoring and safety}

Participation in meditation programmes, even without intensive retreats, could cause or exacerbate negative experiences at rates similar to those occurring in psychotherapy. ${ }^{89}{ }^{90}$ Such negative side effects might carry serious adverse events (eg, psychosis, mania or suicidal ideation) $)^{91} 92$ which, although very infrequent, must be adequately monitored. Therefore, study participants will be requested to report to the psychologist teachers in charge of the groups any sign of deteriorating mental 
or physical health (serious or otherwise) that might arise during the sessions, with special emphasis on possible uncomfortable moments during the exercises and distressing thoughts coming into their minds. ${ }^{93}$ Participants will also be requested to notify the occurrence of such experiences taking place in the period between sessions and/or visits, or any other circumstances that could lead to their consideration of withdrawing from the study. In addition, a checklist will be used to track adverse events during the interventions in order to monitor general potential adverse events such as headaches, dizziness and sleep problems or difficulties across the interventions.

A DMC will be set up, comprising a psychiatrist trial manager, the psychologist teachers in charge of the groups and an independent clinical psychologist familiarised with the management of patients with depressive, anxiety and adjustment disorders. In principle, the DMC will meet three times throughout the trial-after the baseline, post-treatment and follow-up measurementsbut will be available on request at any time to provide support and information to all parties where necessary. The study team will monitor the depression, anxiety and stress independent subscales of the DASS-21 each time it is completed by patients and will inform the DMC in the event of severe or extremely severe scores (z-scores z2). ${ }^{94}$ The DMC will function independently of the sponsors and funders and will oversee and safeguard all trial participant interests, monitoring the overall conduct of the trial and ensuring the safety of patients by systematically checking negative events and reacting to any extreme distress or risk in a participant. In the case of an adverse event emergency, patients will be contacted and encouraged to receive additional help, which will be provided by their corresponding mental health service. Events will be registered/analysed by the DMC to determine whether they are adverse reactions likely associated with the treatment ${ }^{95}$ and whether it is advisable to leave treatment early. Interim analyses are not contemplated in the present study, although the DMC could request them if considered necessary for proper conducting of the trial and/or patient safety.

\section{DISCUSSION}

To the best of our knowledge, this Spanish RCT is the first to directly compare the effectiveness of a protocol based on compassion, such as ABCT, with an MBI, as is the case of the adapted MBSR, in patients suffering from depressive and/or anxious or adjustment disorder with depressive and/or anxious symptomatology in mental health settings. One limitation is that the absence of the full-day silent retreat from the MBSR intervention, and its derived reduction of total in-class contact, might affect the generalisation of the results. However, at the same time, this adaptation might facilitate completion of the programme, while balancing the intensity of both ABCT and MBSR interventions, making them more comparable by matching their application conditions. In addition, all the measurements considered will be self-reported; thus, they will be limited to that type of response. Nevertheless, the results of this study will provide descriptive data that will serve as a reference to drive future research. As we have already mentioned, this study will only be statistically powered to detect the expected large range of differences between groups; thus, small size differences might not show significant effects. However, ES estimation from a direct comparison between the ABCT and MBSR interventions is an area of uncertainty that must be addressed before developing larger studies using more comprehensive and more complex designs. On the other hand, this study will allow us to perform mediating analyses of possible mechanisms of change in ABCT and MBSR group treatments and perform a cost-effectiveness study of these programmes when treating depressive and/ or anxious or adjustment disorders, which are the most prevalent disorders among mental health outpatients in Spain. ${ }^{96}$ This point is of great interest because the Spanish healthcare system generally suffers from a scarcity of resources, such as insufficient length of time spent with each patient and a disproportionate care-providing load. Thus, the demand for care in the Spanish mental health setting does not allow correct implementation of the evidence-based psychotherapeutic treatments of choice that are recommended by the clinical guidelines of reference in terms of duration and periodicity, which might cause a tendency to prescribe medication when not entirely indicated. ${ }^{97} 98$

The lack of funding with which to tackle mental health problems in Spain-which is below the level found in most western European countries-produces both inefficient and inequitable results not only due to the scarce use of group psychological interventions that could bring beneficial solutions to the Spanish health service given the high burden of mental health diseases, but also because of the unequal effect the absence of sufficient resources has on poor people. ${ }^{99}$ How scarce resources are being used is of great importance, and economic evaluations could help to improve decision-making processes. Thus, cost-effective group interventions aimed at treating mild to moderate depressive and/or anxiety or adjustment psychopathological symptoms may be an appropriate solution to reduce the healthcare burden in the Spanish mental health units.

Compassion training might be considered a 'portable therapy' insofar as it is a self-to-self relationship that mimics the compassionate self-to-other relationship of psychotherapy, providing inner strength between sessions and, one hopes, tools for the rest of one's life. ${ }^{42}$ However, due to the state of the art, whether training compassion in mental health units could make a difference in how we understand psychotherapy is a question that requires more evidence. Mindfulness meditation is being increasingly incorporated into mental health programmes, but the compassion construct has not thus far been consistently integrated into widely adopted MBIs. ${ }^{100}$ Results of 
the present study will add relevant information in this respect.

\section{Ethics and dissemination}

All the procedures performed in this study involving human participants will be conducted in accordance with the ethical standards of the 1964 Declaration of Helsinki and its later amendments, the Declaration of Madrid of the World Psychiatric Association and the established requirements for manuscripts submitted to biomedical journals or comparable ethical standards of good clinical practice.

Possible protocol modifications will be decided by the entire research group in consultation with the DMC and will require prior ethics committee and trial registry approvals. Patients who met the inclusion criteria will individually receive a written general overview of the aims and characteristics of the study and interventions by the assessors, who will have sufficient time to answer questions and resolve possible doubts raised to ensure informed consent. Patients will be specifically informed that they will be participating voluntarily and that they can choose to withdraw at any time with a guarantee that they will continue to receive the treatment considered most appropriate by their specialist. After personally informing participants, written informed consent will be obtained from all the participants before they are made aware of the group to which they are to be allocated (an example of the model consent form given to participants is available as online supplementary file 1). For ethical reasons, patients allocated to 'TAU alone' will also be offered the possibility to take part in an adaptation of the mindfulness and compassion programmes at the end of the study after the 6-month follow-up measurement.

Participants' personal information will be stored on a computer server at the research group's headquarters and will be treated in accordance with current EU and Spanish legislation on privacy and data protection. To protect the privacy of participants, all personally identifiable information will be replaced by a randomly assigned username; original data will only be made available to the data manager, who will be the researcher responsible for its supervision. The statistician will be excluded from any information that could facilitate the identification of participants with their corresponding arm throughout the trial. An anonymised and blind-to-arm allocation database copy will be made for use by the statistician, who will conduct the initial primary analysis and, after presenting the corresponding report, the remainder of the analyses. Adverse events and negative side effects will be confidential and only personally identifiable by the data manager and DMC.

All the findings obtained in this study will be submitted to high-impact peer-review journals in the field, and publication authorships will be based on the general recommendations for the conduct, reporting, editing and publication of scholarly work in medical journals. ${ }^{101}$ Study participants will receive a brief personal and comprehensive report on request after the trial conclusion by email. The protocol will be available after its publication. Anonymised participant-level datasets and procedures will be available for replication studies on reasonable request.

\section{Author affiliations}

${ }^{1}$ Primary Care Prevention and Health Promotion Research Network (RedIAPP), Zaragoza, Spain

${ }^{2}$ La Plana University Hospital, Castellón, Spain

${ }^{3}$ Department of Preventive Medicine, Mente Aberta - Brazilian Center for

Mindfulness and Health Promotion, Universidade Federal de São Paulo, UNIFESP,

São Paulo, Brazil

${ }^{4}$ Hospital Israelita Albert Einstein, São Paulo, Brazil

${ }^{5}$ Institute of Health Research of Aragon (IIS), Zaragoza, Spain

Acknowledgements The authors are grateful for the support provided by the Dharamsala Institute of Mindfulness and Psychotherapy of Zaragoza, Spain, as well as the AFDA, and for contributions from patient advisers.

Contributors JG-C and CC-N conceptualised and designed the study. JM-M and CC-N wrote the first draft of the protocol. JM-M developed the statistical analysis plan. MN-G, AL-M, MD, PH-M and AB-S reviewed the manuscript content. All authors read and approved the final manuscript.

Funding The project received funding from DGA group (B17-17R) and the Network for Prevention and Health Promotion in Primary Care (RD16/0007/0005) grant from the Carlos III Health Institute of the Spanish Ministry of Economy and Competitiveness, cofinanced with European Union ERDF funds.

Disclaimer The sources of funding have no influence on the study design; collection, management, analysis and interpretation of the data; or writing of the report and the decision to submit the report for publication; and they will not have authority over any of these activities during the conducting of the trial.

Competing interests None declared.

Patient consent for publication Not required.

Ethics approval Approval was obtained from the Ethics Committee of the General University Hospital of Castellón, Spain (7/2017).

Provenance and peer review Not commissioned; externally peer reviewed.

Data availability statement $A$ data sharing plan has been updated in the corresponding registry record. In accordance with the International Committee of Medical Journal Editors (ICMJE), the data generated by this trial will be made available upon reasonable request to researchers i) who provide a methodologically sound proposal and ii) whose proposed use of the data has been approved by an independent ethical review committee. The data sharing plan includes all of the individual anonymized and completely de-identified participant data collected during the trial, as well as other related documents such as the study protocol, the statistical analysis plan and the data dictionary with descriptive labels. Data will become available immediately following each publication with no end date and for any analytical purpose that is related to achieve aims in the original approved proposal. The database will be encrypted and password protected. Passwords will be provided by the corresponding author to interested researchers that meet the both previously described criteria. The plan and all related documents will be downloadable at: http://doi.org/10.3886/E110024V1.

Open access This is an open access article distributed in accordance with the Creative Commons Attribution Non Commercial (CC BY-NC 4.0) license, which permits others to distribute, remix, adapt, build upon this work non-commercially, and license their derivative works on different terms, provided the original work is properly cited, appropriate credit is given, any changes made indicated, and the use is non-commercial. See: http://creativecommons.org/licenses/by-nc/4.0/.

\section{REFERENCES}

1 Richards D. Prevalence and clinical course of depression: a review. Clin Psychol Rev 2011;31:1117-25.

2 GBD 2015 Disease and Injury Incidence and Prevalence Collaborators. Global, regional, and national incidence, prevalence, and years lived with disability for 310 diseases and injuries, 1990- 
2015: a systematic analysis for the global burden of disease study 2015. Lancet 2016;388:1545-602.

3 World Health Organization. Depression and other common mental disorders: global health estimates. World Heal Organ, 2017: 1-24. http://apps.who.int/iris/bitstream/10665/254610/1/WHO-MSDMER-2017.2-eng.pdf

4 Kuo DC, Tran M, Shah AA, et al. Depression and the suicidal patient. Emerg Med Clin North Am 2015;33:765-78.

5 Wang J, Wu X, Lai W, et al. Prevalence of depression and depressive symptoms among outpatients: a systematic review and meta-analysis. BMJ Open 2017;7:e017173.

6 American Psychiatric Association. Diagnostic and statistical manual of mental disorders. 5th edn. Washington DC: Author, 2013.

7 Bandelow B, Michaelis S. Epidemiology of anxiety disorders in the 21st century. Dialogues Clin Neurosci 2015;17:327-35.

8 Bandelow B, Reitt M, Röver C, et al. Efficacy of treatments for anxiety disorders: a meta-analysis. Int Clin Psychopharmacol 2015;30:183-92.

9 Kessler RC, Berglund P, Demler O, et al. The epidemiology of major depressive disorder: results from the National comorbidity survey replication (NCS-R). JAMA 2003;289:3095-105.

10 Schoevers RA, Deeg DJH, van Tilburg W, et al. Depression and generalized anxiety disorder: co-occurrence and longitudinal patterns in elderly patients. The American Journal of Geriatric Psychiatry 2005;13:31-9.

11 Kessler RC, Merikangas KR, Wang PS. Prevalence, comorbidity, and service utilization for mood disorders in the United States a the beginning of the twenty-first century. Annu Rev Clin Psychol 2007;3:137-58.

12 Carter RM, Wittchen $\mathrm{H}-\mathrm{U}$, Pfister $\mathrm{H}$, et al. One-Year prevalence of subthreshold and threshold DSM-IV generalized anxiety disorder in a nationally representative sample. Depress Anxiety 2001;13:78-88.

13 Maercker A, Lorenz L. Adjustment disorder diagnosis: improving clinical utility. The World Journal of Biological Psychiatry 2018;19:S3-S13.

14 Bachem R, Casey P. Adjustment disorder: a diagnosis whose time has come. J Affect Disord 2018;227:243-53.

15 Reed GM, CORREIA JM, Esparza P, et al. The WPA-WHO global survey of psychiatrists' attitudes towards mental disorders classification. World Psychiatry 2011:10:118-31.

16 Evans SC, Reed GM, Roberts MC, et al. Psychologists' perspectives on the diagnostic classification of mental disorders: results from the WHO-IUPsyS global survey. Int J Psychol 2013;48:177-93.

17 Casey P, Maracy M, Kelly BD, et al. Can adjustment disorder and depressive episode be distinguished? results from Odin. $J$ Affect Disord 2006;92:291-7.

18 Ventevogel P, Pérez-Sales P, Férnandez-Liria A, et al. Integrating mental health care into existing systems of health care: during and after complex humanitarian emergencies. Interv Int J Ment Heal Psychosoc Work Couns Areas Armed Confl 2011;9:195-210.

19 Sundquist J, Ohlsson $\mathrm{H}$, Sundquist $\mathrm{K}$, et al. Common adult psychiatric disorders in Swedish primary care where most mental health patients are treated. BMC Psychiatry 2017;17:235.

20 Baumeister H, Maercker A, Casey P. Adjustment disorder with depressed mood: a critique of its DSM-IV and ICD10 conceptualisations and recommendations for the future. Psychopathology 2009;42:139-47.

21 Servant D, Pelissolo A, Chancharme L, et al. Adjustment disorders with anxiety. Clinical and psychometric characteristics in patients consulting a general practitioner. Encephale 2013;39:347-51.

22 Casey P, Jabbar F, O'Leary E, et al. Suicidal behaviours in adjustment disorder and depressive episode. J Affect Disord 2015:174:441-6.

23 Fernández A, Haro JM, Codony M, et al. Treatment adequacy of anxiety and depressive disorders: primary versus specialised care in Spain. J Affect Disord 2006;96:9-20.

24 Harris MG, Hobbs MJ, Burgess PM, et al. Frequency and quality of mental health treatment for affective and anxiety disorders among Australian adults. Med J Aust 2015;202:185-9.

25 Kladnitski N, Smith J, Allen A, et al. Online mindfulness-enhanced cognitive behavioural therapy for anxiety and depression: outcomes of a pilot trial. Internet Interventions 2018;13:41-50.

26 Huhn M, Tardy M, Spineli LM, et al. Efficacy of pharmacotherapy and psychotherapy for adult psychiatric disorders: a systematic overview of meta-analyses. JAMA Psychiatry 2014;71:706-15

27 Cuijpers P, Gentili C, Baños RM, et al. Relative effects of cognitive and behavioral therapies on generalized anxiety disorder, social anxiety disorder and panic disorder: a meta-analysis. J Anxiety Disord 2016:43:79-89.
28 Sundquist J, Lilja Åsa, Palmér K, et al. Mindfulness group therapy in primary care patients with depression, anxiety and stress and adjustment disorders: randomised controlled trial. $\mathrm{Br} J$ Psychiatry 2015;206:128-35.

29 Hayes SC, Villatte M, Levin M, et al. Open, aware, and active: contextual approaches as an emerging trend in the behavioral and cognitive therapies. Annu Rev Clin Psychol 2011;7:141-68.

30 Lutz A, Jha AP, Dunne JD, et al. Investigating the phenomenological matrix of mindfulness-related practices from a neurocognitive perspective. American Psychologist 2015;70:632-58.

31 Kabat-Zinn J. University of Massachusetts medical Center/ Worcester. stress reduction clinic. full catastrophe living: using the wisdom of your body and mind to face stress, pain, and illness. New York: Bantam Books, 1991

32 Segal ZV, Williams JMG, Teasdale JD. Mindfulness-based cognitive therapy for depression: a new approach to preventing relapse. New York: Guilford Press, 2002

33 Schückher F, Nilsson K. Moderately strong support for mindfulness-based therapies. A literature review of treatment outcome in stress, depression and anxiety. Lakartidningen 2011;108:2020-3

34 Gotink RA, Chu P, Busschbach JJV, et al. Standardised mindfulness-based interventions in healthcare: an overview of systematic reviews and meta-analyses of RCTs. PLoS One 2015;10:e0124344-17.

35 Khoury B, Lecomte T, Fortin G, et al. Mindfulness-based therapy: a comprehensive meta-analysis. Clin Psychol Rev 2013;33:763-71.

36 Wang Y-Y, Li X-H, Zheng W, et al. Mindfulness-based interventions for major depressive disorder: a comprehensive meta-analysis of randomized controlled trials. $J$ Affect Disord 2018;229:429-36.10.1016/j.jad.2017.12.093

37 Williams C, Mercer S. Can mindfulness groups treat common mental health problems? problematic design and short follow-up fails to answer the question. Evid Based Ment Health 2015;18:124.

38 Linardon J, Fairburn CG, Fitzsimmons-Craft EE, et al. The empirical status of the third-wave behaviour therapies for the treatment of eating disorders: a systematic review. Clin Psychol Rev 2017;58:125-40.

39 Feldman C, Kuyken W. Compassion in the landscape of suffering. Contemporary Buddhism 2011;12:143-55.

40 MacBeth A, Gumley A. Exploring compassion: a meta-analysis of the association between self-compassion and psychopathology. Clin Psychol Rev 2012;32:545-52.

41 Montero-Marin J, Kuyken W, Crane C, et al. Self-Compassion and cultural values: a cross-cultural study of Self-Compassion using a Multitrait-Multimethod (MTMM) analytical procedure. Front Psychol 2018;9:2638.

42 Germer CK, Neff KD. Self-Compassion in clinical practice. J Clin Psychol 2013;69:856-67.

$43 \mathrm{Gu}$ J, Strauss C, Bond R, et al. How do mindfulness-based cognitive therapy and mindfulness-based stress reduction improve mental health and wellbeing? A systematic review and meta-analysis of mediation studies. Clin Psychol Rev 2015;37:1-12.

44 Hollis-Walker L, Colosimo K, Mindfulness CK. Mindfulness, selfcompassion, and happiness in non-meditators: a theoretical and empirical examination. Pers Individ Dif 2011;50:222-7.

45 Neff KD, Germer CK. A pilot study and randomized controlled trial of the mindful Self-Compassion program. J Clin Psychol 2013:69:28-44

46 Gilbert P, Procter S. Compassionate mind training for people with high shame and self-criticism: overview and pilot study of a group therapy approach. Clin Psychol Psychother 2006;13:353-79.

47 García-Campayo J, Demarzo M. Mindfulness Y compasión: La nueva revolución. Siglantana: Barcelona, 2015

48 García-Campayo J, Navarro-Gil M, Demarzo M. Attachment-based compassion therapy. Mindfulness \& Compassion 2016;1:68-74.

49 Navarro-Gil M, Lopez-del-Hoyo Y, Modrego-Alarcón M, et al. Effects of Attachment-Based compassion therapy (ABCT) on Selfcompassion and attachment style in healthy people. Mindfulness $2018 ; 13$

50 Homan KJ, Sirois FM. Self-compassion and physical health: exploring the roles of perceived stress and health-promoting behaviors. Health Psychology Open 2017;4.

51 Montero-Marín J, Navarro-Gil M, Puebla-Guedea M, et al. Efficacy of "attachment-based compassion therapy" in the treatment of fibromyalgia: A randomized controlled trial. Front. Psychiatry 2018;8.

52 Moher D, Hopewell S, Schulz KF, et al. Consort 2010 explanation and elaboration: updated guidelines for reporting parallel group randomised trials. BMJ 2010;340:c869. 
53 Chan A-W, Tetzlaff JM, Altman DG, et al. Spirit 2013 statement: defining standard protocol items for clinical trials. Ann Intern Med 2013;158:200-7.

54 First MB, Williams JBW, Karg RS, et al. Structured clinical interview for DSM-5-Research version (SCID-5 for DSM-5, research version; SCID-5-RV). Arlington, Va, American psychiatric association 2015

55 Craig P, Dieppe P, Macintyre S, et al. Developing and evaluating complex interventions: new guidance. UK: Medical Research Council, 2008.

56 Fearon RMP, Roisman GI. Attachment theory: progress and future directions. Current Opinion in Psychology 2017;15:131-6.

57 Santorelli SF, Meleo-Meyers F, Koerbel L. Mindfulness-Based stress reduction (MBSR) authorized curriculum guide. United States, Boston: center for mindfulness in medicine, health care, and Society (CFM) University of Massachusetts medical school, 2017. https://www.umassmed.edu/globalassets/center-for-mindfulness/ documents/mbsr-curriculum-guide-2017.pdf

58 Segal Z, Williams M, Teasdale J. Mindulness-based cognitive therapy for depression: a new approach to preventing relapse. New York, NY: Guilford Press, 2002.

59 Samuelson M, Carmody J, Kabat-Zinn J, et al. Mindfulness-Based stress reduction in Massachusetts correctional facilities. Prison $J$ 2007;87:254-68.

60 Dobkin PL, Hickman S, Monshat K. Holding the heart of Mindfulness-Based stress reduction: balancing fidelity and imagination when adapting MBSR. Mindfulness 2014;5:710-8.

61 Clark LA, Watson D. Tripartite model of anxiety and depression: psychometric evidence and taxonomic implications. J Abnorm Psychol 1991;100:316-36.

62 Lovibond SH, Lovibond PF. Manual for the depression anxiety stress scales. 2nd edn. Sydney N.S.W: Psychology Foundation of Australia, 1995

63 Daza P, Novy DM, Stanley MA, et al. The depression anxiety stress Scale-21: Spanish translation and validation with a Hispanic sample. J Psychopathol Behav Assess 2002;24:195-205.

64 EuroQol Group. EuroQol - a new facility for the measurement of health-related quality of life. Health Policy 1990;16:199-208.

65 Badia X, Roset M, Montserrat S, et al. The Spanish version of EuroQol: a description and its applications. European quality of life scale. Med Clin 1999;112:79-85

66 Baer RA, Smith GT, Hopkins J, et al. Using self-report assessment methods to explore facets of mindfulness. Assessment 2006;13:27-45

67 Neff KD. The development and validation of a scale to measure selfcompassion. Self and Identity 2003;2:223-50.

68 Cebolla A, García-Palacios A, Soler J, et al. Psychometric properties of the Spanish validation of the five facets of mindfulness questionnaire (FFMQ). Eur. J. Psychiat. 2012;26:118-26.

69 Neff KD, Tóth-Király I, Yarnell LM, et al. Examining the factor structure of the Self-Compassion scale in 20 diverse samples: support for use of a total score and six subscale scores. Psychol Assess 2018;31:27-45

70 Garcia-Campayo J, Navarro-Gil M, Andrés E, et al. Validation of the Spanish versions of the long (26 items) and short (12 items) forms of the Self-Compassion scale (scs). Health Qual Life Outcomes 2014;12:4

71 Beecham JK, Knapp MRJ. Costing psychiatric interventions. In: Thornicroft G, ed. Measuring mental health needs. London: Royal Collegues of Psychiatrists, 2001: 200-24.

72 Vazquez-Barquero JL, Gaite L, Cuesta MJ, et al. Spanish version of the CSRI: a mental health cost evaluation interview. Arch Neurobiol 1997;60:171-84

73 Strauss C, Cavanagh K, Oliver A, et al. Mindfulness-based interventions for people diagnosed with a current episode of an anxiety or depressive disorder: a meta-analysis of randomised controlled trials. PLoS One 2014;9:e96110.

74 Boutron I, Moher D, Altman DG, et al. Extending the CONSORT statement to randomized trials of nonpharmacologic treatment: explanation and elaboration. Ann Intern Med 2008:148:295-309.

75 Moher D, Hopewell S, Schulz KF, et al. Consort 2010 explanation and elaboration: updated guidelines for reporting parallel group randomised trials. International Journal of Surgery 2012;10:28-55.

76 Egbewale BE, Lewis M, Sim J. Bias, precision and statistical power of analysis of covariance in the analysis of randomized trials with baseline imbalance: a simulation study. BMC Med Res Methodol 2014;14:49

77 Morris SB. Estimating effect sizes from Pretest-Posttest-Control group designs. Organizational Research Methods 2008;11:364-86.

78 Cohen J. Statistical power analysis for the behavioral sciences (2. Auflage. Hillsdale, NJ: Erlbaum, 1998.

79 Nakagawa S, Johnson PCD, Schielzeth $\mathrm{H}$. The coefficient of determination $R^{2}$ and intra-class correlation coefficient from generalized linear mixed-effects models revisited and expanded. $J$ $R$ Soc Interface 2017;14. doi:10.1098/rsif.2017.0213

80 Jacobson NS, Truax P. Clinical significance: a statistical approach to defining meaningful change in psychotherapy research. $J$ Consult Clin Psychol 1991;59:12-19.

81 White IR, Royston P, Wood AM. Multiple imputation using chained equations: issues and guidance for practice. Stat Med 2011;30:377-99.

82 Kuyken W, Byford S, Taylor RS, et al. Mindfulness-based cognitive therapy to prevent relapse in recurrent depression. J Consult Clin Psychol 2008;76:966-78.

83 Pocock SJ, Assmann SE, Enos LE, et al. Subgroup analysis, covariate adjustment and baseline comparisons in clinical trial reporting: current practiceand problems. Stat Med 2002;21:2917-30

84 Lockhart G, MacKinnon DP, Ohlrich V. Mediation analysis in psychosomatic medicine research. Psychosom Med 2011;73:29-43.

85 Husereau D, Drummond M, Petrou S, et al. Consolidated Health Economic Evaluation Reporting Standards (CHEERS) - Explanation and Elaboration: A Report of the ISPOR Health Economic Evaluation Publication Guidelines Good Reporting Practices Task Force. Value in Health 2013;16:231-50.

86 López-Bastida J, Oliva J, Antoñanzas F, et al. Spanish recommendations on economic evaluation of health technologies. Eur J Health Econ 2010;11:513-20.

87 Oblikue Consulting. Base de Datos de Costes Sanitarios eSALUD Barcelona, 2014. Available: http://www.oblikue.com/bddcostes

88 Feise RJ. Do multiple outcome measures require $p$-value adjustment? BMC Med Res Methodol 2002;2:8.

89 Lindahl JR, Fisher NE, Cooper DJ, et al. The varieties of contemplative experience: a mixed-methods study of meditation-related challenges in Western Buddhists. PLoS One 2017;12:e0176239.

90 Crawford MJ, Thana L, Farquharson L, et al. Patient experience of negative effects of psychological treatment: results of a national survey. British Journal of Psychiatry 2016;208:260-5.

91 Van Dam NT, van Vugt MK, Vago DR, et al. Reiterated concerns and further challenges for mindfulness and meditation research: a reply to Davidson and Dahl. Perspect Psychol Sci 2018;13:66-9.

92 Kuijpers $\mathrm{HJH}$, van der Heijden FMMA, Tuinier S, et al. MeditationInduced psychosis. Psychopathology 2007;40:461-4.

93 Galante J, Dufour G, Benton A, et al. Protocol for the mindful student study: a randomised controlled trial of the provision of a mindfulness intervention to support university students' well-being and resilience to stress. BMJ Open 2016;6:e012300.

94 Lovibond PF, Lovibond SH. Manual for the depression anxiety stress scales. 2nd ed. Sydney: Psychology Foundation, 1995.

95 Nhs National Institute for health research. clinical trials toolkit 2016, 2 October 2017. Available: http://www.ct-toolkit.ac.uk/

96 Gutierrez-Fraile M, Garcia-Calvo C, Prieto R, et al. Mental disorders in psychiatric outpatients in Spain. Actas Esp Psiquiatr 2011;39:349-55.

97 Girón M, Beviá B, Medina E, et al. [Quality of the physician-patient relation and results of clinical encounters in primary care in Alicante: a study with focal groups]. Rev Esp Salud Publica 2002;76:561-75.

98 Gonzalez S, Gonzalez M, Iglesias MC, et al. Psychological treatments features and outcomes in Spanish public mental health centres. International Journal of Psychology and Psychological Therapy 2014;14:17-32.

99 Salvador-Carulla L, Garrido M, McDaid D, et al. Financing mental health care in Spain: context and critical issues. Eur. J. Psychiat. 2006;20:29-44

100 Wielgosz J, Goldberg SB, Kral TRA, et al. Mindfulness meditation and psychopathology. Annu Rev Clin Psychol 2019;15:285-316.

101 International Committee of medical Journal editors (ICMJE). Available: http://www.icmje.org [Accessed 2 November 2017] 